\title{
Proteins involved in the endoplasmic reticulum stress are key players in synovitis of osteoarthritis, chronic pyrophosphate arthropathy and rheumatoid arthritis, and correlate with the histological score
}

Dominique de Seny ( $\square$ ddeseny@chuliege.be)

Laboratory of rheumatology, GIGA Research, CHU Liège

Elettra Bianchi

CHU de Liege - Hopital du Sart Tilman

Dominique Baiwir

Universite de Liege

Gaël Cobraiville

Centre hospitalier universitaire de Liege

Charlotte Collin

Universite de Liege

Mégane Deliège

Universite de Liege

Marie-Joëlle Kaiser

Centre hospitalier universitaire de Liege

Gabriel Mazzucchelli

Universite de Liege

Jean-Philippe Hauzeur

Centre hospitalier universitaire de Liege

Philippe Delvenne

Centre hospitalier universitaire de Liege

Michel Malaise

Centre hospitalier universitaire de Liege

Research article

Keywords: proteomic, synovitis, endoplasmic reticulum, biomarker, chaperone, S100A8, S100A9, complement

Posted Date: March 24th, 2020 
DOI: https://doi.org/10.21203/rs.3.rs-18867/v1

License: (c) (1) This work is licensed under a Creative Commons Attribution 4.0 International License. Read Full License 
1 Proteins involved in the endoplasmic reticulum stress are key players in synovitis of

2 osteoarthritis, chronic pyrophosphate arthropathy and rheumatoid arthritis, and 3 correlate with the histological score

4

5 Dominique de Seny (1), Elettra Bianchi (2), Dominique Baiwir (3), Gaël Cobraiville (1), 6 Charlotte Collin (1), Mégane Deliège (1), Marie-Joëlle Kaiser (1), Gabriel Mazzucchelli (4), 7 Jean-Philippe Hauzeur (1), Philippe Delvenne (2), Michel G. Malaise (1).

\section{Affiliation:}

10 (1) Laboratory of Rheumatology, GIGA Research, CHU Liege, 4000 Liege, Belgium.

11 (2) Department of Pathology, GIGA Research, CHU Liege, 4000 Liège, Belgium

12 (3) GIGA Proteomics Facility, University of Liege, 4000 Liege, Belgium

(4) Mass Spectrometry Laboratory, MolSys Unit Research, University of Liege, 4000 Liege,

14 Belgium

\section{Corresponding author:}

17 Dominique de Seny

18 Rheumatology department, Tour GIGA, +2

19 CHU, 4000 Liège

20 Belgium

21 office-phone : +3243662474

22 e-mail : ddeseny@chuliege.be

24 Running Headline: Proteins of ER stress in synovitis

25 Conflict of interest: All authors have nothing to disclose 


\section{$1 \quad$ Abstract}

2 Background: It is now well recognized that osteoarthritis (OA) synovial membrane presents

3 inflammatory components. The aim of this work is to provide evidence that similar

4 inflammatory mechanisms exist among the three pathologies presenting an inflammatory

5 gradient: OA, chronic pyrophosphate arthropathy (CPPA) and rheumatoid arthritis (RA).

6 Methods: Synovial biopsies of OA $(n=9)$, CPPA $(n=7)$ and RA $(n=8)$ patients were first

7 characterized by a histological score based on synovial hyperplasia and infiltration of

8 lymphocytes, plasma cells, polymorphonuclear and macrophages. All biopsies were also

9 analyzed by 2D-nano-UPLC-ESI-Q-Orbitrap for protein identification and quantification.

10 Protein levels were correlated with the histological score.

11 Results: Histological score was in the range of 3 to 8 for OA, 5 to 13 for CPPA and 12 to 17

12 for RA. Of the 4336 proteins identified by mass spectrometry, 51 proteins were selected for 13 their strong correlation $(\mathrm{p}<0.001)$ with the histological score of which 11 proteins (DNAJB11, 14 CALR, ERP29, GANAB, HSP90B1, HSPA1A, HSPA5, HYOU1, LMAN1, PDIA4, and TXNDC5) were involved in the endoplasmic reticulum (ER) stress. Protein levels of S100A8 and S100A9 were significantly higher in RA compared to OA (for both) or to CPPA (for 17 S100A8 only) and also significantly correlated with the histological score. Eighteen 18 complement component proteins were identified, but only one (complement C1q 19 subcomponent, subunit B and C) was weakly correlated with the histological score.

Conclusions: This study highlights the inflammatory gradient existing between OA, CPPA and

21 RA synovitis either at the protein level or at the histological level. Inflamed synovitis was characterized by the overexpression of ER stress proteins.

24 Key words: proteomic, synovitis, endoplasmic reticulum, biomarker, chaperone, S100A8, 


\section{Abbreviation}

2 2D-nano-UPLC-ESI-Q-Orbitrap : 2 dimensional-nano-ultra performance liquid

3 chromatography-electrospay ionization-Q-Orbitrap

4 CALR: calreticulin

5 CPPA: chronic pyrophosphate arthropathy

6 CRP: C-reactive protein

7 DAMPS: damage-associated molecular patterns

8 ER: endoplasmic reticulum

9 ERAD: ER-associated degradation

10 GRP78: Glucose-Regulated Protein 78 kDa

11 LC-MS/MS: Liquid chromatography-tandem mass spectrometry

12 LFQ: label free quantitative

13 MRI: magnetic resonance imaging

14 OA: osteoarthritis

15 PMN: polymorphonuclear cells

16 RA: rheumatoid arthritis

17 TLR: toll-like receptors

18 TXNDC5: Thioredoxin domain-containing protein 5

19 UPR: unfolded protein response

20 US: ultrasonography 


\section{INTRODUCTION}

Osteoarthritis (OA) was for long considered as a degenerative cartilage disease for which synovitis was only visualized in the late stages and considered as secondary to mechanic aggression of bone and cartilage degradation. However, several observations demonstrated that synovitis could appear even in the early stages of OA. Synovium can also acquire an "inflammatory" phenotype in OA with similar characteristics than those observed in rheumatoid arthritis (RA) [i.e. synovial lining and villous hyperplasia, infiltration by macrophages and lymphocytes, neo-angiogenesis and fibrosis](1,2). Using magnetic resonance imaging (MRI), Roemer et al. noted a synovitis in $96.3 \%$ of knee joints with effusion and in $70 \%$ of knee joint without effusion(3). We previously published by using ultrasonography (US) examination that $53.7 \%(322 / 600)$ of patients with painful knee OA had no sign of inflammation whereas $2.7 \%(16 / 600)$ had synovitis alone, $14.2 \%(85 / 600)$ had both synovitis and effusion and 29.5\% (177/600) had joint effusion alone(4). US knee synovitis and US joint effusion were significantly associated with a more severe radiological grade (KellgrenLawrence grade $\geq 3$ ) and a moderate-important joint effusion at clinical examination(4). Further, several other studies have confirmed the correlation between synovitis area observed by MRI and specific histologic features of synovitis(5).

Two major pathways at least can explain the development of synovitis: activation of toll-like receptors (TLR) and activation of the complement cascade(1). Endogenous "damageassociated molecular patterns" (DAMPS) can activate the innate immune response through TLRs recognition promoting pro-inflammatory mediators secretion $(6,7)$.

Activation of the complement cascade induces complement deposits sparsely found in the synovium of OA patients. Deposits of synovial complement components were only observed during acute OA flare but not during chronic OA(8). More recently, proteomic 
analyses of OA synovial fluids $(9,10)$ and transcriptomic studies of OA synovial

2 membranes(10) confirmed the expression and activation of complement in joints(11). Proteomic analysis of synovial tissue was rarely performed(12,13) and none was compared to the histological pattern of synovium. In this work, we compared protein profiles generated by a proteomic study to the histological features of synovial biopsies obtained from

6 patients with OA, chronic pyrophosphate arthropathy (CPPA) or RA. It unraveled an increased gradient of inflammation and synovial lining hyperplasia among the three pathologies both at the protein and histological levels.

\section{METHODS}

\section{Patients and synovial tissue}

All experiments undertaken with patient material complied with the regulations and ethical guidelines of the CHU of Liege, Belgium. Synovial biopsies of OA ( $n=9)$, CPPA $(n=7)$ and RA patients $(n=8)$ were obtained by needle arthroscopy from affected knees. For each patient, 3 synovial fragments were stored at $-80^{\circ} \mathrm{C}$ until used for proteomic study. Three other fragments were also processed for formalin fixation $(24 \mathrm{~h})$ using a standard procedure and were embedded in paraffin for microscopic examination of the hematoxylin and eosin (H\&E) stained sections and histological scoring.

\section{Histological score}

Hematoxylin eosin stained sections were scored as previously done for routine clinical analysis and randomly analyzed as described in Tak et al(14). Briefly, all areas of each biopsy

23 section were examined by trained anatomopathologists (P.D. and E. B.). Histological features

24 included synovial hyperplasia and the degree of infiltration of lymphocytes, plasma cells or 25 polymorphonuclear cells (PMN), separately. The TAK score(14) was slightly modified: a) 
1 synovial hyperplasia was scored on a five-point scale (0-4) instead of a four-point scale (0-3)

2 because synovitis hallmark in RA as in OA is proliferation and hyperplasia of the lining cells(2) and b) PMN infiltration was scored on a four-point scale (0-3) instead of a five-point scale (04) because PMN infiltration is less intense than any other mononuclear cells infiltration. Macrophage infiltration was also determined by using immunohistochemistry with an antiCD68 antibody [anti-CD68 (KP-1) Primary Antibody, Ventana Medical System], and the CD68 expression was scored separately using a semi-quantitative 4 scale score $(0-3)$ with 0 for no infiltration, 1, 2 and 3 for respectively mild, moderate and severe infiltration using a pre-defined atlas(15). The histological score was determined by the sum of the components, as for other methods(14,15), leading to a maximum of 18 .

\section{LC-MS/MS analysis for proteomic analysis}

The biopsies were weighted $(5 \mathrm{mg})$ and resuspended in $300 \mu \mathrm{L}$ of RIPA buffer in the presence of complete and phospho stop solutions. The biopsies were then disrupted using a biswitch ( $+60 \mathrm{mg}$ of $\mu$ beads) for cycles of $30 \mathrm{sec}$ high speed and $30 \mathrm{sec}$ low speed at $4^{\circ} \mathrm{C}$, during 2X15 min, to allow proteins dissolution in the buffer. The protein concentration of each sample was determined using the RCDC protein assay kit according to manufacturer recommendations (BioRad, Hercules, CA, USA). For each sample, $15 \mu \mathrm{g}$ of protein were diluted in ammonium bicarbonate $(50 \mathrm{mM})$ to get a protein concentration of $0.5 \mu \mathrm{g} / \mu \mathrm{L}$. The proteins were then reduced (DTT), alkylated with iodoacetamide and precipitated using the 2D clean-up kit (GE Healthcare, Belgium). The protein pellets were then resuspended in ammonium bicarbonate (50 $\mathrm{mM}$ ) at a concentration of $0.5 \mu \mathrm{g} / \mu \mathrm{L}$ and digested with trypsin. For each sample, $3.5 \mu \mathrm{g}$ of peptides were desalted using Ziptip C18 (Millipore Corp., Billerica, MA, USA) according to the manufacturer's instructions. The eluted fractions were then dried by speed-vac. Dry pellets were stored at $-20^{\circ} \mathrm{C}$ until used for analysis. Before injection into the 2D-nano-UPLC system, 
$12.5 \mu \mathrm{g}$ of the digested proteins were resuspended in $9 \mu \mathrm{L}$ of $100 \mathrm{mM}$ ammonium formate solution

2 adjusted to $\mathrm{pH} 10$. A standard MassPREPTM (MPDS mixture) digestion mixture (Waters Corp.,

3 Milford, USA) which contains a mixture of yeast enolase (ENO1, P00924), rabbit glycogen

4 phosphorylase b (GPB, P00489), yeast alcohol dehydrogenase (ADH, P00330) and bovine 5 serum albumin (BSA, P02769) was spiked in each sample at a quantity of 150 fmoles of ADH 6 digest per injection.

7

All samples were injected on a 2D-nanoAquity UPLC (Waters, Corp., Milford, USA) coupled online with a ESI-Q-Orbitrap (Q Exactive, Thermo Fisher Scientific) in positive ion mode. Briefly, the liquid chromatography approach used was a 2-dimensional method (2D-LC) comprising 3 steps of 180 min. The 3 steps were carried out on a high $\mathrm{pH}$ column with increasing percentage of acetonitrile. The eluted peptides were then injected onto a low $\mathrm{pH}$ column for which each step consists of a 5 min gradient from $99 \%$ buffer $\mathrm{A}\left(\mathrm{A}=\mathrm{H}_{2} \mathrm{O}, 0.1 \%\right.$ formic acid, $\mathrm{B}=$ acetonitrile) to $93 \%$ of $\mathrm{A}$ followed by a gradient of $135 \mathrm{~min}$ from $93 \%$ of $\mathrm{A}$ to $65 \%$ of $\mathrm{A}$. The acquisition method was a TopN-MSMS where $\mathrm{N}$ was set to 12 , meaning that the spectrometer acquired one Full MS spectrum, selected the 12 most intense peaks in this spectrum (singly charged precursors excluded) and recorded a Full MS2 spectrum of each of these 12 compounds. The parameters for MS spectrum acquisition were: mass range from 400 to $1750 \mathrm{~m} / \mathrm{z}$, resolution of 70000 , automated gain control (AGC) target of $10^{6}$ or maximum injection time of $200 \mathrm{~ms}$. The parameters for MS2 spectrum acquisition were: isolation window of $2.0 \mathrm{~m} / \mathrm{z}$, collision energy (NCE) of 25 , resolution of 17500 , AGC target of $10^{5}$ or maximum injection time of 50ms. The database searches were performed by the software MaxQuant ver.1.5.2.8. Protein identifications were considered as significant if these proteins were identified with at least 2 peptides including at least one unique peptide per protein considering a false positive discovery rate $(\mathrm{FDR})<0.01$. 
2 Data analysis

Epidemiological data of the 3 groups (OA, CPPA and RA) were compared using the

4 Kruskal Wallis test with the posthoc test of Dunn's for continuous variables and Chi square test

5 for qualitative variables. Comparison of two-by-two groups for K\&L was performed with Mann

6 Whitney test.

For MS/MS values, Maxquant analysis leads to the identification and calculation of the normalized protein intensities. The label free quantitative (LFQ) intensities can be directly compared between samples (for a given protein) and can be imported in Perseus software (version 1.5.5.0) for statistical differential analysis. Only proteins identified in 7 biopsies of at

11 least one of the three groups (OA, CPPA and RA) were considered for further analysis. 1871 12 proteins were selected accordingly. LFQ intensities were Log2 transformed for all statistical analyses. Correlation coefficients were obtained using Pearson test after verifying that all values passed the D'Agostino normality test. Multiple-comparison test (ANOVA) was used for comparing protein intensities of the three groups [OA, CPPA and RA]. DAVID Bioinformatics resources 6.8 was used for the identification of KEGG pathways. STRING 10.5 was used for functional protein association network.

\section{RESULTS}

\section{Characteristics of patients}

Clinical and biologic data are summarized in Table 1. Parameters related to age, gender and BMI were not statistically different between OA, CPPA and RA patients. Severity of OA and CPPA was defined according to the Kellgren Lauwrence (K\&L) grade(16) and was not statistically different between the two groups. Histological score was significantly different between the 3 groups $(\mathrm{P}$ value $=0.0003)$ and was higher in $\mathrm{RA}$ compared to $\mathrm{OA}(\mathrm{P}<0.001)$ or 
1 CPPA $(\mathrm{P}<0.05)$ but not different between OA and CPPA groups according to the posthoc test.

2 CRP values exceeding the normal range were observed in $20 \%, 40 \%$ and $90 \%$ of OA, CPPA

3 and RA patients, respectively; all patients being untreated by corticosteroids or any disease

4 modifying anti-rheumatic drugs, including biologics.

6 Histological score

Histological score was in the range of 3 to 8 for OA, 5 to 13 for CPPA and 12 to 17 for

RA, which represents a continuum for the inflammatory process of the synovial membrane through the 24 biopsies, the least severe being the OA group in contrast to CPPA (medium score) and RA (highest score) (Table 1, Figure 1A). Of note, there is an overlap between the 3

11 pathologies, and some patients with OA have already a medium high inflammatory score.

12 Synovial hyperplasia and infiltration of lymphocytes were observed in all samples (Figure 1A and 1B), whereas plasmocytic infiltration was observed in only 3/9 OA (33\%), 3/7 (43\%) CPPA and 8/8 RA synovitis, and PMN infiltration in 0/9 OA, in 1/7 (14\%) CPPA and in 6/8 (75\%) RA synovitis (Figure 1A and 1B). Macrophage infiltration was present in 8/9 (89\%) of OA, in 5/7 (71\%) of CPPA and in 8/8 RA synovitis (Figure 1A and 1C).

\section{Proteomics analysis}

Proteins were extracted and digested from the 24 biopsies for proteomic analysis by mass spectrometry (MS/MS). 4336 proteins were identified by MS/MS, but only 1871 proteins were selected for their significant identification in 7 biopsies of at least one of the three groups (OA, CPPA or RA). The 1871 proteins intensities were then correlated with their corresponding histological score. Fifty-one proteins presented a statistically significant correlation with a P value being at least $<0.001$ (Table 2), including 39 proteins with a positive correlation $(\mathrm{r}>0.63$ ) and 12 proteins with a negative correlation $(r<-0.65)$ (Table 2$)$. Some proteins of Table 2 are 
1 illustrated in Figure 2 for the three groups, as well as correlation parameters between MS/MS

$2 \log 2$ protein intensities and respective histological score. Highlighted grey lines of Table 2

3 feature 31 proteins detected in the entire set of the 24 biopsies, including 27 proteins being

4 positively correlated with the histological score and 4 proteins being negatively correlated 5 (Table 2).

We also confirmed that DAMP proteins, S100A8 and S100A9, were detected in the 24 synovial membrane biopsies and that their protein levels were strongly increased in RA compared to OA biopsies (ANOVA, Tuckey post hoc test $\mathrm{P}<0.01$ ). Only S100A8 but not S100A9 protein levels were discriminant between CPPA and RA groups $(\mathrm{P}<0.01)$. As expected, a strong correlation between $\mathrm{S} 100 \mathrm{~A} 8$ and $\mathrm{S} 100 \mathrm{~A} 9$ protein levels $(\mathrm{r}=0.95, \mathrm{P}<0.0001)$ was determined among the 3 groups.

DAVID analysis was performed on the 51 biomarkers to highlight their functional classifications (Figure 3A). The pathway entitled "protein processing in endoplasmic reticulum (ER)" was selected and weighted in the balance for $22 \%$ (Figure $3 \mathrm{~A}$ ). In this pathway, 11 proteins were identified: DNAJB11, CALR, ERP29, GANAB, HSP90B1, HSPA1A, HSPA5, HYOU1, LMAN1, PDIA4, and TXNDC5 (Figure 3B) and were connected by the STRING protein-protein interaction network (see red writings in Figure 3C). Statistically significant positive correlations were confirmed and summarized in Figure 3D, except for HSPA1A that was negatively correlated to the 10 others. Out of that observation, we calculated the ratio of HSPA1A to TXNDC5 protein levels that was negatively correlated $(\mathrm{r}=-0.6, \mathrm{P}=0.002)$ with the histological score (Figure 3E). Although DAVID analysis did not highlight a pathway centered on the alarmins S100A8 and S100A9, both proteins were significantly correlated with almost all 50 other proteins selected in Table 2, except for PARP1 and CRTAC1 for S100A8 or S100A9, and GANAB and GNB2L1 for S100A9 (Table 3). The same 39 proteins as in Table 2 
1 were positively correlated with S100A8 or S100A9, as well as the 12 proteins that were negatively correlated (Table 3).

A special attention was drawn to the status of complement component protein levels: 18 have been identified among the 1871 proteins retained (Additional file 1). Only complement C1q subunits B and C were slightly significantly correlated with the histological score.

\section{DISCUSSION}

OA synovitis share at a lower degree many common features with RA synovitis including abnormal synoviocytes proliferation, leukocytes infiltration and angiogenesis. In our study, synovial hyperplasia and lymphocyte infiltration were observed in all samples. Macrophages infiltration was detected in all biopsies except for 2 OA and 1 CPPA synovitis. Plasmocytes infiltration was observed in all RA and in $33-43 \%$ of non-RA biopsies, whereas PMN infiltration was present in $75 \%$ of RA biopsies and in $0-10 \%$ of non-RA biopsies. The histological score highlighted an inflammatory continuum through the 24 biopsies with an overlap between the 3 studied pathologies in agreement with other publications(1,2,15). It emphasizes the absence of a unique pattern for each studied disease and the heterogeneity of cell infiltration, either quantitatively and qualitatively. Synovitis is composed of various inflammatory cells and proliferating synoviocytes that induce the secretion of classical inflammatory mediators but also the secretion of thousands of proteins that represents the dark proteome, a Gordian knot that can only be unraveled by a tissue proteomic analysis.

To the best of our knowledge, this is the first proteomic study of human synovitis for which proteins levels were compared to their corresponding histological score. DAVID and STRING analyses highlighted 11 proteins involved in the endoplasmic reticulum pathway: DNAJB11, CALR, ERP29, GANAB, HSP90B1, HSPA1A, HSPA5, HYOU1, LMAN1, PDIA4, and TXNDC5. All these proteins were detected in the 24 biopsies supporting the relevance of 
1 their identifications. How can we connect ER proteins to the inflammatory process inside synovitis? Endoplasmic reticulum (ER) is an intracellular organelle playing a major role in proper proteins folding through the activation of several chaperone proteins, including protein disulfide isomerase (PDI), ERP29, the Hsp70 family member Glucose-Regulated Protein 78 $\mathrm{kDa}(\mathrm{GRP} 78 / \mathrm{BiP})(H S P A 5)$, and calreticulin (CALR). However, despite the function of these

6 chaperones, the success rate for proper folding is often quite low. Incompletely folded proteins 7 are forced to be removed from cells by a process called UPR (unfolded protein response) activated along with the ER-associated degradation (ERAD), enhancing protein degradation by the proteasome. Some cellular disturbances such as nutrient deprivation, hypoxia or loss of calcium homeostasis can disrupt ER efficiency and lead to the accumulation of unfolded 11 proteins enhancing a stress response in the ER. Under normal conditions, GRP78/BiP (HSPA5) maintains the canonical UPR regulators (IRE1 $\alpha$, PERK and ATF6) in an inactivated form, while upon pathological conditions, it dissociates from the three UPR proteins inducing UPR activation(17). Contribution of the ER stress in RA pathogenesis has been recently described(17). Inflammation and ER stress work together by driving inflammatory cells to produce pro-inflammatory cytokines but also to enhance FLS proliferation and angiogenesis $(17,18)$. Further, synovial hyperplasia is linked to chronic inflammation and joint destruction(1). GRP78/BiP has been localized predominantly in the lining but also sublining layers of RA (more pronounced) and OA synovium(18). Downregulation of GRP78/BiP increases apoptosis of RA FLS and conversely its overexpression 21 prevents cells from apoptotic death induced by an ER stressor(18). Selective abrogation of GRP78/BiP blunts activation of NF- $\kappa \mathrm{B}$ and protect mice from collagen arthritis(19). ERdj3 (DNAJB11) acts as a component with other co-chaperone proteins SDF2 and 24 SDF2L1 in the GRP78/BiP chaperone cycle to prevent the aggregation of misfolded proteins(20) and regulates GRP78/BiP occupancy in living cells(21). Two other ER 
1 chaperones, such as GRP94/endoplasmin (HSP90B1) and calreticulin (CALR) contribute to the

2 autoimmune process in different ways. Under physiologic conditions, GRP94/endoplasmin

3 optimizes the function of B cells by chaperoning TLRs(22). Indeed, GRP94/endoplasmin

4 ablation in B cells attenuated antibody production in the context of TLR stimulation(22). Under

5 pathologic conditions, GRP94/endoplasmin translocates to the cell surface and extracellular

6 space and could function as an autoantigen to induce autoantibodies and enhance immune

7 responses(23). GRP94/endoplasmin may also act as an endogenous ligand of TLR2 to promote

8 chronic inflammation(23). GRP94/endoplasmin induces the transcription of TLR2, TNF- $\alpha$ and

9 IL8 but not TLR4 in synovial macrophages(24). GRP94/endoplasmin is highly expressed in the

10 lining and sublining layers of RA synovium correlating with lining thickness (lining) and the

11 inflammatory score (sublining)(24). Its expression was also detected in control OA

12 synovium(24). A recent study demonstrated that the upregulation of Bcl-XL and Mcl-1

13 expression in RA FLS by calreticulin promoted apoptosis resistance of RA FLS(25).

14 Calreticulin promotes angiogenesis by activating nitric oxide signaling pathway in $\operatorname{RA}(26)$.

15 Further, soluble calreticulin can induce the expression of pro-inflammatory cytokines by 16 macrophages(27). Calreticulin was previously detected by another proteomic study focusing on 17 formalin-fixed paraffin-embedded (FFPE) synovial tissues provided from OA and RA 18 tissues(12).

Hypoxia-upregulated protein 1 (HYOU1) or GRP170 is co-regulated and associated

20 with two other chaperones GRP78/BiP and GRP94/endoplasmin, suggesting their coordinated

21 activity in the maintenance of protein homeostasis(28). HYOU1 presents an important 22 cytoprotective role in hypoxia-induced cellular perturbation(29) and can contribute to cell

23 survival when ER is under stress. However, surface or extracellular HYOU1 exerts documented

24 immunoregulatory activities in some immunopathologies but its role in rheumatic diseases 25 remains unknown(30). In addition to its function as a "danger" molecule alerting the immune 
1 system of tissue damage, the extracellular HYOU1 has also the capacity of amplifying the

2 inflammatory response triggered by microbial signals and possibly by DAMPs(30). HYOU1

3 also promoted pulmonary fibrosis in mice by increasing pulmonary levels of TGF- $\beta 1$ and

4 myofibroblasts(31).

Thioredoxin domain-containing protein $5(T X N D C 5)$ is a protein disulfide isomerase

6 with clear pro-inflammatory properties. TXNDC5 contributes to abnormal RA FLS

7 proliferation, migration and IL-6 production by inhibiting IGFBP1 expression(32).

8 Downregulation of TXNDC5 could contribute to RA FLS antiangiogenic and proapoptotic

9 features through the suppression of CXCL10 and TRAIL(33). Further, TXNDC5 synergizes

10 with heat shock cognate 70 protein (HSC70) to exacerbate the inflammatory phenotype of RA

11 FLS through NF- $\kappa$ B signaling(34). TXNDC5 expression was increased in synovial tissues of

12 RA patients compared to $\mathrm{OA}$ as identified by a proteomic study(13) or by immunochemistry(35). Further, elevated levels were found in the synovial fluid and serum of RA patients(35).

The role of ERp29 (ERP29) seems controversial in the literature regarding to apoptosis. It protects cells such as neurons from apoptosis(36) whereas it sensitizes some others such as cancer cells(37). Murine macrophages upon interaction with heat-inactivated Candida albicans unravel an anti-inflammatory response with the overexpression of ERp29(38). Protein disulfide-isomerase-A4 (PDIA4) mediates resistance to cisplatin-induced cell death in lung adenocarcinoma(39). PDIA4 mRNAs were significantly increased in patients' inflamed colonic mucosa compared to uninflamed mucosa and controls(40). Protein ERGIC-53 (LMAN1) is a type I transmembrane protein that is located at the ER, ER-Golgi Intermediate Compartment (ERGIC) and cis-Golgi. Protein ERGIC-53 facilitates transport of several cargo proteins

24 including factors critical to the coagulation cascade from the ER to Golgi(41). Interaction of 
1 apoptosis(42). GANAB is a key glycoprotein quality control protein in ER removing glucose residues from immature glycoproteins(43). Although ERp29, PDIA4, Protein ERGIC-53 and GANAB proteins were highly positively correlated in our work with the histological score, their presence and their role in the pathophysiology of synovitis have not yet been described. As for other chaperones, the heat shock $70 \mathrm{kDa}$ protein 1A/1B or Hsp72 (HSPA1A) can

6 be released by normal cell under stress or by damaged cell, but unlike most of other chaperones, HSPA1A displays anti-inflammatory properties. Recombinant human HSPA1A suppresses the production of pro-inflammatory cytokines in RA FLS by inhibition of NF- $\mathrm{B}$ pathway and decreases collagen-induced arthritis in mice(44). Interestingly, in our study, HSPA1A levels were significantly lower in RA than in OA or CPPA synovium and negatively correlated with the histological score as well as with the 10 other aforementioned protein levels of the ER pathway suggesting a defective anti-inflammatory response in favor of a pro-inflammatory one under the control of ER proteins. S100A8 and S100A9 were expressed in the 24 biopsies and positively correlated with the histological score. Further, they were almost correlated to all 51 proteins highlighted for their correlation with the histological score. S100A8 and S100A9 proteins are Ca2+ binding proteins constitutively expressed by neutrophils and monocytes. They are well-known DAMPs proteins participating to leukocyte recruitment and cytokines secretion and are highly expressed in many inflammatory conditions. They were detected in serum(45), synovial fluid(46) and joint tissue $(12,13)$ of RA patients. In the OA synovium, they are mainly produced by M-1 like macrophages and slightly by M-2 like macrophages but not by FLS, inducing inflammatory cytokines and MMPs expression(47). S100A8 and S100A9 are actively involved in the thickening of the intimal layer and in the development of joint destruction in murine collagenase-induced OA but not in destabilized medial meniscus-induced OA(48). Further, S100A8 and S100A9 levels in the OA synovitis significantly correlate with synovial lining 
1 thickness and cellularity in the subintima(48). Gene and protein expression of S100A9 were

2 increased in inflamed areas as compared to normal area of human OA synovitis(49).

Eighteen complement component proteins were identified but none except complement

4 C1q was positively correlated to the histological score. These findings are in contrast with two

5 other proteomic analyses $(9,10)$ performed in OA synovial fluids but not in tissue. Indeed,

6 Gobezie et al. identified C3 as a discriminant marker exhibiting a sensitivity of $90 \%$ and a

7 specificity of $85 \%$ in OA synovial fluids(9). Similarly, we also identified previously increased

8 levels of C3f fragment in synovial fluid of OA patients (11). Complement components can be

9 delivered from blood by ultrafiltration. Numerous publications mentioned that complement

10 components could be produced by synovial tissue cells(50). However, in our study, complement

11 component levels in OA, CPPA and RA were not correlated to the histological score and were

12 not statistically elevated among the three pathologies, suggesting that complement cascade was

13 further playing a role in synovial fluids and not in synovial tissue.

\section{CONCLUSION}

This study highlights the continuum existing between OA, CPPA and RA synovitis both at the protein and the histological score levels. These two levels are connected giving pathophysiological relevance of the proteomic synovium analysis. Pannus development in these diseases is characterized by overexpression of many proteins involved in the ER stress, mostly chaperones and co-chaperones. All studied ER proteins except HSPA1A exhibit pro-

21 inflammatory properties when they are exposed on the cell membrane or secreted outside

22 favoring inflammatory cytokine production as well as proliferation and migration of FLS. Five proteins (HSPA5, HSB90B1, CALR, TXNDC5 and HSPA1A) have been previously identified in the RA synovium and to a lesser extent in the OA synovium. Six proteins (DNAJB11, HYOUI, ERp29, PDIA4, LMAN1 and GANAB) have never been described in RA or in OA synovium, 
1 and none in CPPA synovium. These data confirm an important role for these chaperones and

2 co-chaperones of the ER pathway in the pathophysiology of RA, but more importantly strongly

3 suggest a similar unknown pattern in the pathophysiology of OA and CPPA. S100A8 and

4 S100A9 were correlated to the histological score and to most of highlighted proteins in Table

5 2. Complement components seem to behave independently from the histological score. We

6 have also highlighted the negative correlation between the histological score and the

7 HSPA1A/TXNDC5 ratio, which fits with the capacity of TXNDC5 to exacerbate the

8 inflammatory phenotype of FLS(34). This proteomic analysis suggests the need for future

9 developments such as: 1) the identification of other pathways including other proteins that are

10 not correlated with the histological score, and 2) the characterization of protein clusters

11 correlated with each type of cells infiltrating the pannus as well as with FLS proliferative

12 capacity. This tool may allow to develop a molecular classification of complex rheumatic 13 diseases.

DECLARATIONS

16

Ethics approval and consent to participate

18 All experiments undertaken with patient material complied with the regulations and ethical 19 guidelines of the CHU of Liege, Belgium.

20 Consent for publication: Not applicable

21 Availability of data and materials: The datasets used and/or analyzed during the current study

22 are available from the corresponding author on reasonable request.

23 Competing interests: We declare that we have no competing of interest.

24 Funding: This study was supported by the "Fond d'Investissement pour la Recherche 25 Scientifique" (FIRS), CHU Liège, Belgium. 


\section{Authors' contributions:}

2 All authors were involved in drafting and revision of the manuscript and all authors approved

3 the final version to be published.

4 Study conception and design: de Seny D, Malaise MG

5 Acquisition of data: Bianchi E, Baiwir D, Cobraiville G, Collin C, Deliège M, Kaiser MJ,

6 Mazzucchelli G, Hauzeur JP

7 Analysis and interpretation of data: de Seny D, Delvenne P, Malaise MG

8 Acknowledgements: We thank the GIGA Proteomics Facility, ULiege.

\section{REFERENCES}

12 1. Scanzello CR, Goldring SR. The role of synovitis in osteoarthritis pathogenesis. Bone. 2012 Aug;51(2):249-57.

2. Mathiessen A, Conaghan PG. Synovitis in osteoarthritis: current understanding with therapeutic implications. Arthritis Res Ther. 2017 Feb 2;19(1):18.

3. Roemer FW, Kassim Javaid M, Guermazi A, Thomas M, Kiran A, Keen R, et al. Anatomical distribution of synovitis in knee osteoarthritis and its association with joint effusion assessed on non-enhanced and contrast-enhanced MRI. Osteoarthr Cartil. 2010 Oct;18(10):1269-74.

4. D'Agostino MA, Conaghan P, Le Bars M, Baron G, Grassi W, Martin-Mola E, et al. EULAR report on the use of ultrasonography in painful knee osteoarthritis. Part 1: prevalence of inflammation in osteoarthritis. Ann Rheum Dis. 2005 Dec;64(12):17039. between synovitis detected on enhanced-magnetic resonance imaging and a histological 
analysis with a patient-oriented outcome measure for Japanese patients with end-stage knee osteoarthritis receiving joint replacement surgery. Clin Rheumatol. 2010 Oct;29(10):1185-90.

6. Gómez R, Villalvilla A, Largo R, Gualillo O, Herrero-Beaumont G. TLR4 signalling in osteoarthritis-finding targets for candidate DMOADs. Nat Rev Rheumatol. 2015 Mar 16;11(3):159-70.

7. de Seny D, Cobraiville G, Charlier E, Neuville S, Esser N, Malaise D, et al. Acute-phase serum amyloid a in osteoarthritis: regulatory mechanism and proinflammatory properties. PLoS One. 2013;8(6):e66769.

8. Konttinen YT, Ceponis A, Meri S, Vuorikoski A, Kortekangas P, Sorsa T, et al. Complement in acute and chronic arthritides: assessment of $\mathrm{C} 3 \mathrm{c}, \mathrm{C} 9$, and protectin (CD59) in synovial membrane. Ann Rheum Dis. 1996 Dec;55(12):888-94.

9. Gobezie R, Kho A, Krastins B, Sarracino DA, Thornhill TS, Chase M, et al. High abundance synovial fluid proteome: distinct profiles in health and osteoarthritis. Arthritis Res Ther. 2007;9(2):R36.

10. Ritter SY, Subbaiah R, Bebek G, Crish J, Scanzello CR, Krastins B, et al. Proteomic analysis of synovial fluid from the osteoarthritic knee: comparison with transcriptome analyses of joint tissues. Arthritis Rheum. 2013 Apr;65(4):981-92.

11. de Seny D, Sharif M, Fillet M, Cobraiville G, Meuwis M-A, Marée R, et al. Discovery and biochemical characterisation of four novel biomarkers for osteoarthritis. Ann Rheum Dis. 2011 Jun;70(6):1144-52.

12. Hayashi J, Kihara M, Kato H, Nishimura T. A proteomic profile of synoviocyte lesions microdissected from formalin-fixed paraffin-embedded synovial tissues of rheumatoid arthritis. Clin Proteomics. 2015;12(1):20.

13. Chang X, Cui Y, Zong M, Zhao Y, Yan X, Chen Y, et al. Identification of proteins with 
increased expression in rheumatoid arthritis synovial tissues. J Rheumatol. 2009 May;36(5):872-80.

14. Tak PP, Thurkow EW, Daha MR, Kluin PM, Smeets TJM, Meinders AE, et al. Expression of adhesion molecules in early rheumatoid synovial tissue. Clin Immunol Immunopathol. 1995 Dec;77(3):236-42.

15. Najm A, le Goff B, Venet G, Garraud T, Amiaud J, Biha N, et al. IMSYC immunologic synovitis score: A new score for synovial membrane characterization in inflammatory and non-inflammatory arthritis. Joint Bone Spine [Internet]. 2019 Jan;86(1):77-81.

16. Kellgren JH, Lawrence JS. Radiological assessment of osteo-arthrosis. Ann Rheum Dis. 1957 Dec;16(4):494-502.

17. Rahmati M, Moosavi MA, McDermott MF. ER Stress: A Therapeutic Target in Rheumatoid Arthritis? Trends Pharmacol Sci. 2018;39(7):610-23.

18. Yoo S-A, You S, Yoon H-J, Kim D-H, Kim H-S, Lee K, et al. A novel pathogenic role

21. Guo F, Snapp EL. ERdj3 regulates BiP occupancy in living cells. J Cell Sci. 2013 Mar

20. Fujimori T, Suno R, Iemura S-I, Natsume T, Wada I, Hosokawa N. Endoplasmic reticulum proteins SDF2 and SDF2L1 act as components of the BiP chaperone cycle to prevent protein aggregation. Genes Cells. 2017 Aug;22(8):684-98. 
via chaperoning integrin and TLR but not immunoglobulin. Blood. 2008 Aug $15 ; 112(4): 1223-30$.

23. Huang Q-Q, Pope RM. The role of glycoprotein 96 in the persistent inflammation of rheumatoid arthritis. Arch Biochem Biophys. 2013 Feb 1;530(1):1-6.

24. Huang Q-Q, Sobkoviak R, Jockheck-Clark AR, Shi B, Mandelin AM, Tak PP, et al. Heat shock protein 96 is elevated in rheumatoid arthritis and activates macrophages primarily via TLR2 signaling. J Immunol. 2009 Apr 15;182(8):4965-73.

25. Jiao Y, Ding H, Huang S, Liu Y, Sun X, Wei W, et al. Bcl-XL and Mcl-1 upregulation by calreticulin promotes apoptosis resistance of fibroblast-like synoviocytes via activation of PI3K/Akt and STAT3 pathways in rheumatoid arthritis. Clin Exp Rheumatol. 2018;36(5):841-9.

26. Ding $\mathrm{H}$, Hong $\mathrm{C}$, Wang $\mathrm{Y}$, Liu J, Zhang N, Shen C, et al. Calreticulin promotes angiogenesis via activating nitric oxide signalling pathway in rheumatoid arthritis. Clin Exp Immunol. 2014 Nov;178(2):236-44.

27. Duo C-C, Gong F-Y, He X-Y, Li Y-M, Wang J, Zhang J-P, et al. Soluble calreticulin induces tumor necrosis factor- $\alpha$ (TNF- $\alpha$ ) and interleukin (IL)-6 production by macrophages through mitogen-activated protein kinase (MAPK) and NFkB signaling pathways. Int J Mol Sci. 2014 Feb 20;15(2):2916-28.

28. Lin HY, Masso-Welch P, Di YP, Cai JW, Shen JW, Subjeck JR. The 170-kDa glucoseregulated stress protein is an endoplasmic reticulum protein that binds immunoglobulin. Mol Biol Cell. 1993 Nov;4(11):1109-19.

29. Ozawa K, Kuwabara K, Tamatani M, Takatsuji K, Tsukamoto Y, Kaneda S, et al. 150$\mathrm{kDa}$ oxygen-regulated protein (ORP150) suppresses hypoxia-induced apoptotic cell death. J Biol Chem. 1999 Mar 5;274(10):6397-404.

30. Zuo D, Subjeck J, Wang X-Y. Unfolding the Role of Large Heat Shock Proteins: New 
Insights and Therapeutic Implications. Front Immunol. 2016;7(MAR):75.

31. Tanaka K, Shirai A, Ito Y, Namba T, Tahara K, Yamakawa N, et al. Expression of 150$\mathrm{kDa}$ oxygen-regulated protein (ORP150) stimulates bleomycin-induced pulmonary fibrosis and dysfunction in mice. Biochem Biophys Res Commun. 2012 Sep $7 ; 425(4): 818-24$.

32. Li J, Xu B, Wu C, Yan X, Zhang L, Chang X. TXNDC5 contributes to rheumatoid arthritis by down-regulating IGFBP1 expression. Clin Exp Immunol. 2018;192(1):8294.

33. Xu B, Li J, Wu C, Liu C, Yan X, Chang X. CXCL10 and TRAIL Are Upregulated by TXNDC5 in Rheumatoid Arthritis Fibroblast-like Synoviocytes. J Rheumatol. 2018 Mar;45(3):335-40.

34. Wang L, Dong H, Song G, Zhang R, Pan J, Han J. TXNDC5 synergizes with HSC70 to exacerbate the inflammatory phenotype of synovial fibroblasts in rheumatoid arthritis through NF-кB signaling. Cell Mol Immunol. 2018;15(7):685-96.

35. Chang X, Zhao Y, Yan X, Pan J, Fang K, Wang L. Investigating a pathogenic role for TXNDC5 in rheumatoid arthritis. Arthritis Res Ther. 2011 Jul 29;13(4):R124.

36. Zhang Y-H, Belegu V, Zou Y, Wang F, Qian B-J, Liu R, et al. Endoplasmic Reticulum Protein 29 Protects Axotomized Neurons from Apoptosis and Promotes Neuronal Regeneration Associated with Erk Signal. Mol Neurobiol. 2015 Aug;52(1):522-32.

37. Zhang D, Richardson DR. Endoplasmic reticulum protein 29(ERp29): An emerging role in cancer. Int J Biochem Cell Biol. 2011 Jan;43(1):33-6.

38. Martínez-Solano L, Reales-Calderón JA, Nombela C, Molero G, Gil C. Proteomics of RAW 264.7 macrophages upon interaction with heat-inactivated Candida albicans cells unravel an anti-inflammatory response. Proteomics. 2009 Jun;9(11):2995-3010.

39. Tufo G, Jones AWE, Wang Z, Hamelin J, Tajeddine N, Esposti DD, et al. The protein 
disulfide isomerases PDIA4 and PDIA6 mediate resistance to cisplatin-induced cell death in lung adenocarcinoma. Cell Death Differ. 2014 May;21(5):685-95.

40. Negroni A, Prete E, Vitali R, Cesi V, Aloi M, Civitelli F, et al. Endoplasmic reticulum stress and unfolded protein response are involved in paediatric inflammatory bowel disease. Dig Liver Dis. 2014 Sep;46(9):788-94.

41. Zhang B, Kaufman RJ, Ginsburg D. LMAN1 and MCFD2 form a cargo receptor complex and interact with coagulation factor VIII in the early secretory pathway. J Biol Chem. 2005 Jul 8;280(27):25881-6.

42. Nelson TJ, Alkon DL. Protection against beta-amyloid-induced apoptosis by peptides interacting with beta-amyloid. J Biol Chem. 2007 Oct 26;282(43):31238-49.

43. Pelletier MF, Marcil A, Sevigny G, Jakob CA, Tessier DC, Chevet E, et al. The heterodimeric structure of glucosidase II is required for its activity, solubility, and localization in vivo. Glycobiology. 2000;10(8):815-27.

44. Luo X, Zuo X, Mo X, Zhou Y, Xiao X. Treatment with recombinant Hsp72 suppresses collagen-induced arthritis in mice. Inflammation. 2011 Oct;34(5):432-9.

45. de Seny D, Fillet M, Ribbens C, Marée R, Meuwis M-A, Lutteri L, et al. Monomeric calgranulins measured by SELDI-TOF mass spectrometry and calprotectin measured by ELISA as biomarkers in arthritis. Clin Chem. 2008 Jun;54(6):1066-75.

46. Baillet A. [S100A8, S100A9 and S100A12 proteins in rheumatoid arthritis]. La Rev Med interne. 2010 Jun;31(6):458-61.

47. van den Bosch MH, Blom AB, Schelbergen RF, Koenders MI, van de Loo FA, van den Berg WB, et al. Alarmin S100A9 Induces Proinflammatory and Catabolic Effects Predominantly in the M1 Macrophages of Human Osteoarthritic Synovium. J Rheumatol. 2016 Oct;43(10):1874-84.

48. van Lent PLEM, Blom AB, Schelbergen RFP, Slöetjes A, Lafeber FPJG, Lems WF, et 
al. Active involvement of alarmins S100A8 and S100A9 in the regulation of synovial activation and joint destruction during mouse and human osteoarthritis. Arthritis Rheum. 2012 May;64(5):1466-76.

49. Lambert C, Dubuc J-E, Montell E, Vergés J, Munaut C, Noël A, et al. Gene expression pattern of cells from inflamed and normal areas of osteoarthritis synovial membrane. Arthritis Rheumatol (Hoboken, NJ). 2014 Apr;66(4):960-8.

50. Neumann E, Barnum SR, Tarner IH, Echols J, Fleck M, Judex M, et al. Local production of complement proteins in rheumatoid arthritis synovium. Arthritis Rheum. 2002 Apr;46(4):934-45.

\section{FIGURE LEGENDS}

Figure 1: Histological scoring of synovial biopsies A) Classification of OA, CPPA and RA synovial biopsies $(n=24)$ according to the histological score based on the following criteria: synovial hyperplasia (hs, $0-4)$, infiltration of lymphocytes (ly, $0-4)$, plasmocytes $(\mathrm{pl}, 0-4)$, polynuclear neutrophils (po, $0-3$ ) and macrophages (CD68, $0-3)$. B) Histological representation of hematoxylin eosin stained sections for synovial hyperplasia and infiltration of lymphocytes/plasmocytes and polynuclear neutrophils in one OA, CPPA or RA biopsy. C) Immunohistochemistry using anti-CD68 antibody showing macrophage infiltration in OA, CPPA and RA synovial biopsies.

\section{Figure 2: Distribution of protein intensities among the 3 groups (OA, CPPA and RA) and} correlation with the histological score

Illustration of some proteins from Table 2 for which $\log 2$ intensities obtained by MS/MS are represented among the three groups (OA, CPPA and RA) and statistically correlated to the histological score. 
2 Figure 3: ER stress proteins detected in the inflamed synovial membrane: A) DAVID 3 analysis performed on the 51 biomarkers highlighted for their significant correlation to the

4 histological score, for their functional classifications. The pathway entitled "protein processing 5 in endoplasmic reticulum (ER)" was selected. B) Proteins involved in the ER pathway 6 according to DAVID analysis. C) STRING protein-protein interaction among the 51 proteins 7 highlighted in Table 2. Red writings indicate proteins involved in the ER network according to 8 DAVID analysis. D) Correlation parameters between the 11 proteins involved in the ER 9 according to DAVID. E) Negative correlation between HSPA1A and TXNDC5 protein levels.

11 Additional file 1: Correlation parameters between complement components and the 12 histological score

13 Representation of complement components intensities obtained by MS/MS among the three 14 groups (OA, CPPA and RA). * represent P-values $<0.05$; ns = not significant 15 16 
1 Table 1: Patients description

2 Clinical and pathological characteristics of patients with osteoarthritis (OA), chronic

3 pyrophosphate arthropathy (CPPA) or rheumatoid arthritis (RA)

\begin{tabular}{lllll}
\hline & OA & CPPA & RA & P value \\
\hline $\mathrm{n}$ & & & & \\
Age [median (interval)] & 9 & 7 & 5 & \\
\% of woman & $55(36-89)$ & $65(50-74)$ & $62 \%(5 / 8)$ & 0.44 \\
BMI [median (interval)] & $88 \%(8 / 9)$ & $71 \%(5 / 7)$ & $24.4(16.4-33.9)$ & 0.4 \\
K\&L score [median (interval)] & $32.2(17.6-41.9)$ & $24.2(22-33)$ & $/$ & 0.6 \\
Histological inflammatory score & $3(0-4)$ & $2(0-4)$ & $14(12-17)$ & 0.0003 \\
Anti-CCP (positive \%) & $4(3-8)$ & $5(5-13)$ & & 0.002 \\
Rheumatoid factor (positive \%) & & & $60 \%$ & 0.032 \\
CRP (Positive \%) & $0 \%$ & $0 \%$ & $90 \%$ & 0.020 \\
ESR (Positive \%) & $20 \%$ & $0 \%$ & $60 \%$ & 0.01 \\
\end{tabular}

$\mathrm{OA}=$ osteoarthritis; $\mathrm{CPPA}=$ chronic pyrophosphate arthropathy; $\mathrm{RA}$ = rheumatoid arthritis;

$\mathrm{BMI}=$ Body mass index; $\mathrm{K} \& \mathrm{~L}=$ Kellgren and Lauwrence score;

anti-CCP $=$ anti-cyclic citrullinated peptide; $\mathrm{CRP}=\mathrm{C}$ reactive protein; $\mathrm{ESR}=$ erythrocyte sedimentation rate 
1 Table 2: Correlation parameters between protein intensities and histological score

2 Intensities of 51 proteins quantified by MS/MS were significantly correlated to the histological

3 score. $\mathrm{n}=$ number of biopsies for which the protein was detected; $\mathrm{r}=$ coefficient correlation

4 (Pearson test). P-values are statistically significant $<0.001$.

\begin{tabular}{|c|c|c|c|c|c|}
\hline Gene ID & Prot ID & Prot name & n & $\mathbf{r}$ & P-value \\
\hline LSP1 & P33241 & Lymphocyte-specific protein 1 & 16 & 0.83 & $<0.0001$ \\
\hline MZB1 & Q8WU39 & Marginal zone B- and B1-cell-specific protein & 15 & 0.80 & 0.0004 \\
\hline MANF & P55145 & Mesencephalic astrocyte-derived neurotrophic factor & 23 & 0.79 & $<0.0001$ \\
\hline EML4 & Q9HC35 & Echinoderm microtubule-associated protein-like 4 & 20 & 0.78 & $<0.0001$ \\
\hline LAP3 & P28838 & Cytosol aminopeptidase & 24 & 0.77 & $<0.0001$ \\
\hline DNAJB11 & Q9UBS4 & DnaJ homolog subfamily B member 11 & 24 & 0.77 & $<0.0001$ \\
\hline DEFA1 & P59665 & Neutrophil defensin 1 & 21 & 0.76 & $<0.0001$ \\
\hline ERP29 & P30040 & Endoplasmic reticulum resident protein 29 & 24 & 0.75 & $<0.0001$ \\
\hline IDH2 & P48735 & Isocitrate dehydrogenase [NADP], mitochondrial & 24 & 0.75 & $<0.0001$ \\
\hline LCP1 & P13796 & Plastin-2 & 24 & 0.74 & $<0.0001$ \\
\hline TXNDC5 & Q8NBS9 & Thioredoxin domain-containing protein 5 & 24 & 0.73 & $<0.0001$ \\
\hline HSP9OB1 & P14625 & Endoplasmin or glucose-related protein 94 (GRP94) & 24 & 0.73 & $<0.0001$ \\
\hline CALR & P27797 & Calreticulin & 24 & 0.73 & $<0.0001$ \\
\hline PRDX4 & Q13162 & Peroxiredoxin-4 & 24 & 0.73 & $<0.0001$ \\
\hline SRP72 & 076094 & Signal recognition particle subunit SRP72 & 22 & 0.73 & 0.0001 \\
\hline HSPA5 & P11021 & $78 \mathrm{kDa}$ glucose-regulated protein (GRP78) or BiP & 24 & 0.72 & $<0.0001$ \\
\hline ARHGDIB & P52566 & Rho GDP-dissociation inhibitor 2 & 24 & 0.72 & $<0.0001$ \\
\hline PDIA4 & P13667 & Protein disulfide-isomerase A4 & 24 & 0.72 & $<0.0001$ \\
\hline TAPBP & 015533 & Tapasin & 18 & 0.72 & 0.0009 \\
\hline CORO1A & P31146 & Coronin-1A & 24 & 0.71 & $<0.0001$ \\
\hline S100A8 & P05109 & Protein $\mathrm{S} 100-\mathrm{A} 8$ & 24 & 0.71 & 0.0001 \\
\hline CTSS & P25774 & Cathepsin S & 22 & 0.69 & 0.0003 \\
\hline CTSZ & Q9UBR2 & Cathepsin Z & 24 & 0.69 & 0.0002 \\
\hline MNDA & P41218 & Myeloid cell nuclear differentiation antigen & 23 & 0.69 & 0.0003 \\
\hline LMNB1 & P20700 & Lamin-B1 & 24 & 0.69 & 0.0002 \\
\hline TUBA4A & P68366 & Tubulin alpha-4A chain & 24 & 0.68 & 0.0002 \\
\hline PMM2 & 015305 & Phosphomannomutase 2 & 20 & 0.68 & 0.0009 \\
\hline CNPY2 & Q9Y2B0 & Protein canopy homolog 2 & 24 & 0.68 & 0.0003 \\
\hline PTPRC & P08575 & Receptor-type tyrosine-protein phosphatase $\mathrm{C}$ & 23 & 0.68 & 0.0004 \\
\hline S100A9 & P06702 & Protein S100-A9 & 24 & 0.68 & 0.0003 \\
\hline LMAN1 & P49257 & Protein ERGIC-53 & 24 & 0.68 & 0.0003 \\
\hline EEF1G & P26641 & Elongation factor 1-gamma & 24 & 0.67 & 0.0004 \\
\hline STAT1 & P42224 & Signal transducer and activator of transcription 1-alpha/beta & 23 & 0.66 & 0.0007 \\
\hline GBP1 & P32455 & Interferon-induced guanylate-binding protein 1 & 24 & 0.65 & 0.0005 \\
\hline GNB2L1 & P63244 & Guanine nucleotide-binding protein subunit beta-2-like 1 & 24 & 0.65 & 0.0006 \\
\hline PARP1 & P09874 & Poly [ADP-ribose] polymerase 1 & 24 & 0.64 & 0.0007 \\
\hline PFN1 & P07737 & Profilin-1 & 24 & 0.64 & 0.0008 \\
\hline HYOU1 & Q9Y4L1 & Hypoxia up-regulated protein 1 (GRP170) & 24 & 0.63 & 0.0009 \\
\hline GANAB & Q14697 & Neutral alpha-glucosidase $A B$ & 24 & 0.63 & 0.001 \\
\hline$T N X B$ & P22105 & Tenascin-X & 23 & -0.65 & 0.0008 \\
\hline CRTAC1 & Q9NQ79 & Cartilage acidic protein 1 & 22 & -0.66 & 0.0009 \\
\hline HSPA1A & PODMV8 & Heat shock $70 \mathrm{kDa}$ protein $1 \mathrm{~A} / 1 \mathrm{~B}$ (HSP70-1) & 24 & -0.66 & 0.0005 \\
\hline SPTBN1 & Q01082 & Spectrin beta chain. non-erythrocytic 1 & 24 & -0.67 & 0.0003 \\
\hline SNTB2 & Q13425 & Beta-2-syntrophin & 24 & -0.67 & 0.0004 \\
\hline LEMD2 & Q8NC56 & LEM domain-containing protein 2 & 21 & -0.67 & 0.0008 \\
\hline LMNB2 & Q03252 & Lamin-B2 & 24 & -0.68 & 0.0003 \\
\hline$C K B$ & P12277 & Creatine kinase B-type & 20 & -0.68 & 0.0009 \\
\hline GPX3 & P22352 & Glutathione peroxidase 3 & 23 & -0.69 & 0.0002 \\
\hline$C P Q$ & Q9Y646 & Carboxypeptidase Q & 18 & -0.75 & 0.0004 \\
\hline SYNE3 & Q6ZMZ3 & Nesprin-3 & 23 & -0.75 & $<0.0001$ \\
\hline SCARA5 & Q6ZMJ2 & Scavenger receptor class A member 5 & 15 & -0.85 & $<0.0001$ \\
\hline
\end{tabular}


1 Table 3: Correlation parameters between S100A8/S100A9 and the 50 protein intensities

2 correlated to the histological score

3 Alarmins S100A8 and S100A9 were both correlated to the 50 other protein intensities obtained

4 by MS/MS and correlated to the histological score. $r=$ coefficient correlation (Pearson test).

$5 \quad *, * *, * * *$ represent $\mathrm{P}$-values $<0.05 ; 0.01$ and 0.001 , respectively. $\mathrm{ns}=$ not significant

\begin{tabular}{|c|c|c|c|c|}
\hline & S100A8 & & S100A9 & \\
\hline & Pearson $r$ & $P$ value & Pearson $\mathrm{r}$ & $P$ value \\
\hline Protein S100-A9 & 0.9545 & $* * *$ & 0.9545 & $* * *$ \\
\hline Neutrophil defensin & 0.9131 & $* * *$ & 0.8637 & $* * *$ \\
\hline Lymphocyte-specific protein 1 & 0.8527 & $* * *$ & 0.8197 & $* * *$ \\
\hline Coronin-1A & 0.8417 & $* * *$ & 0.8041 & $* * *$ \\
\hline Plastin-2 & 0.837 & $* * *$ & 0.8021 & $* * *$ \\
\hline Cathepsin Z & 0.8266 & $* * *$ & 0.7937 & $* * *$ \\
\hline Myeloid cell nuclear differentiation antigen & 0.8118 & $* * *$ & 0.7771 & $* * *$ \\
\hline Tapasin & 0.7384 & $* * *$ & 0.6698 & $* *$ \\
\hline Profilin-1 & 0.7348 & $* * *$ & 0.6748 & $* * *$ \\
\hline Mesencephalic astrocyte-derived neurotrophic factor & 0.7223 & $* * *$ & 0.7417 & $* * *$ \\
\hline Cathepsin S & 0.7125 & $* * *$ & 0.6177 & $* *$ \\
\hline Receptor-type tyrosine-protein phosphatase C & 0.707 & $* * *$ & 0.643 & $* * *$ \\
\hline Rho GDP-dissociation inhibitor 2 & 0.6958 & $* * *$ & 0.6217 & $* *$ \\
\hline Calreticulin & 0.68 & $* * *$ & 0.6088 & $* *$ \\
\hline Cytosol aminopeptidase & 0.679 & $* * *$ & 0.6264 & $* *$ \\
\hline Marginal zone B- and B1-cell-specific protein & 0.667 & $* *$ & 0.7098 & $* *$ \\
\hline Elongation factor 1-gamma & 0.6522 & $* * *$ & 0.5827 & $* *$ \\
\hline Lamin-B1 & 0.6505 & $* * *$ & 0.587 & $* *$ \\
\hline Echinoderm microtubule-associated protein-like 4 & 0.6346 & $* *$ & 0.5933 & $* *$ \\
\hline Phosphomannomutase 2 & 0.614 & $* *$ & 0.6091 & $* *$ \\
\hline Endoplasmic reticulum resident protein 29 & 0.5962 & ** & 0.644 & $* * *$ \\
\hline Peroxiredoxin-4 & 0.578 & $* *$ & 0.5787 & $* *$ \\
\hline Protein canopy homolog 2 & 0.5758 & $* *$ & 0.6744 & $* * *$ \\
\hline Signal transducer and activator of transcription 1-alpha/beta & 0.5704 & $* *$ & 0.5427 & $* *$ \\
\hline DnaJ homolog subfamily B member 11 & 0.5504 & ** & 0.5635 & $* *$ \\
\hline Interferon-induced guanylate-binding protein 1 & 0.5373 & $* *$ & 0.566 & $* *$ \\
\hline Hypoxia up-regulated protein 1 (GRP170) & 0.5357 & $* *$ & 0.5298 & $* *$ \\
\hline 78 kDa glucose-regulated protein (GRP78) or BiP & 0.5233 & $* *$ & 0.5043 & $*$ \\
\hline Endoplasmin & 0.5164 & ** & 0.4804 & $*$ \\
\hline Isocitrate dehydrogenase [NADP], mitochondrial & 0.5118 & * & 0.4164 & $*$ \\
\hline Protein disulfide-isomerase A4 & 0.5076 & $*$ & 0.4499 & $*$ \\
\hline Protein ERGIC-53 & 0.4971 & $*$ & 0.4479 & $*$ \\
\hline Tubulin alpha-4A chain & 0.4941 & $*$ & 0.4951 & $*$ \\
\hline Neutral alpha-glucosidase $A B$ & 0.483 & * & 0.396 & ns \\
\hline Guanine nucleotide-binding protein subunit beta-2-like & 0.4502 & $*$ & 0.3353 & ns \\
\hline Signal recognition particle subunit SRP72 & 0.4444 & * & 0.4308 & $*$ \\
\hline Thioredoxin domain-containing protein 5 & 0.4049 & * & 0.4182 & $*$ \\
\hline Poly [ADP-ribose] polymerase 1 & 0.3561 & ns & 0.2772 & ns \\
\hline Cartilage acidic protein 1 & -0.3355 & ns & -0.3434 & ns \\
\hline Heat shock 70 kDa protein 1A/1B (HSP70-1) & -0.5366 & $* *$ & -0.5838 & $* *$ \\
\hline LEM domain-containing protein 2 & -0.6254 & ** & -0.5722 & $* *$ \\
\hline Nesprin-3 & -0.6296 & $* *$ & -0.6015 & $* *$ \\
\hline Tenascin-X & -0.6321 & $* *$ & -0.5679 & $* *$ \\
\hline Spectrin beta chain, non-eryththrocytic 1 & -0.6884 & $* * *$ & -0.6734 & $* * *$ \\
\hline Scavenger receptor class A member 5 & -0.7069 & $* *$ & -0.6619 & $* *$ \\
\hline Carboxypeptidase Q & -0.7156 & $* * *$ & -0.7019 & $* *$ \\
\hline Lamin-B2 & -0.7414 & $* * *$ & -0.7293 & $* * *$ \\
\hline Glutathione peroxidase 3 & -0.7475 & $* * *$ & -0.7667 & $* * *$ \\
\hline Beta-2-syntrophin & -0.7522 & $* * *$ & -0.8171 & $* * *$ \\
\hline Creatine kinase B-type & -0.7555 & $* * *$ & -0.7136 & $* * *$ \\
\hline
\end{tabular}




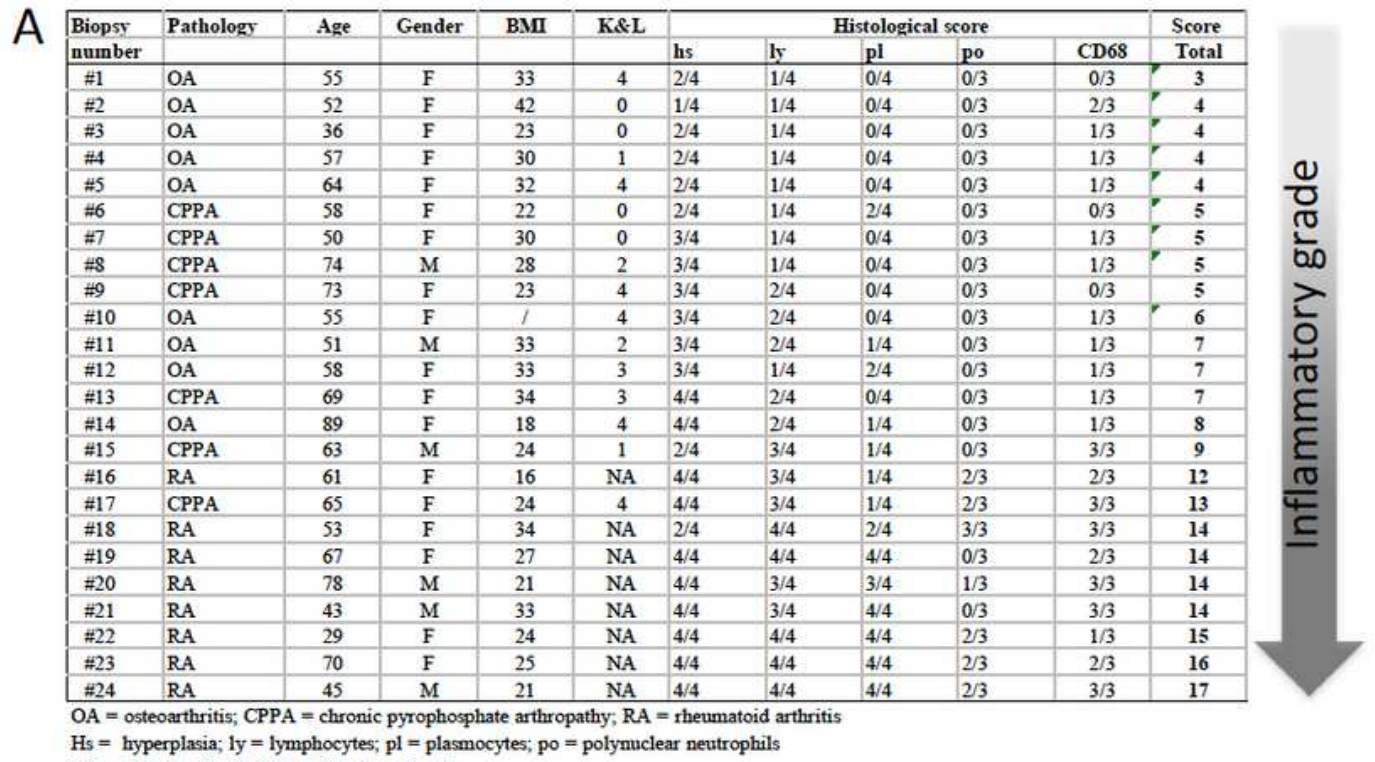

B

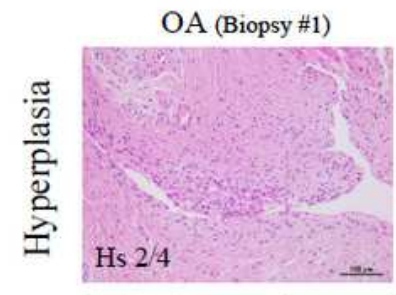

CPPA (Biopsy \#17)

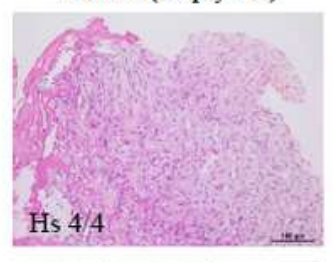

RA (Biopsy \#18)
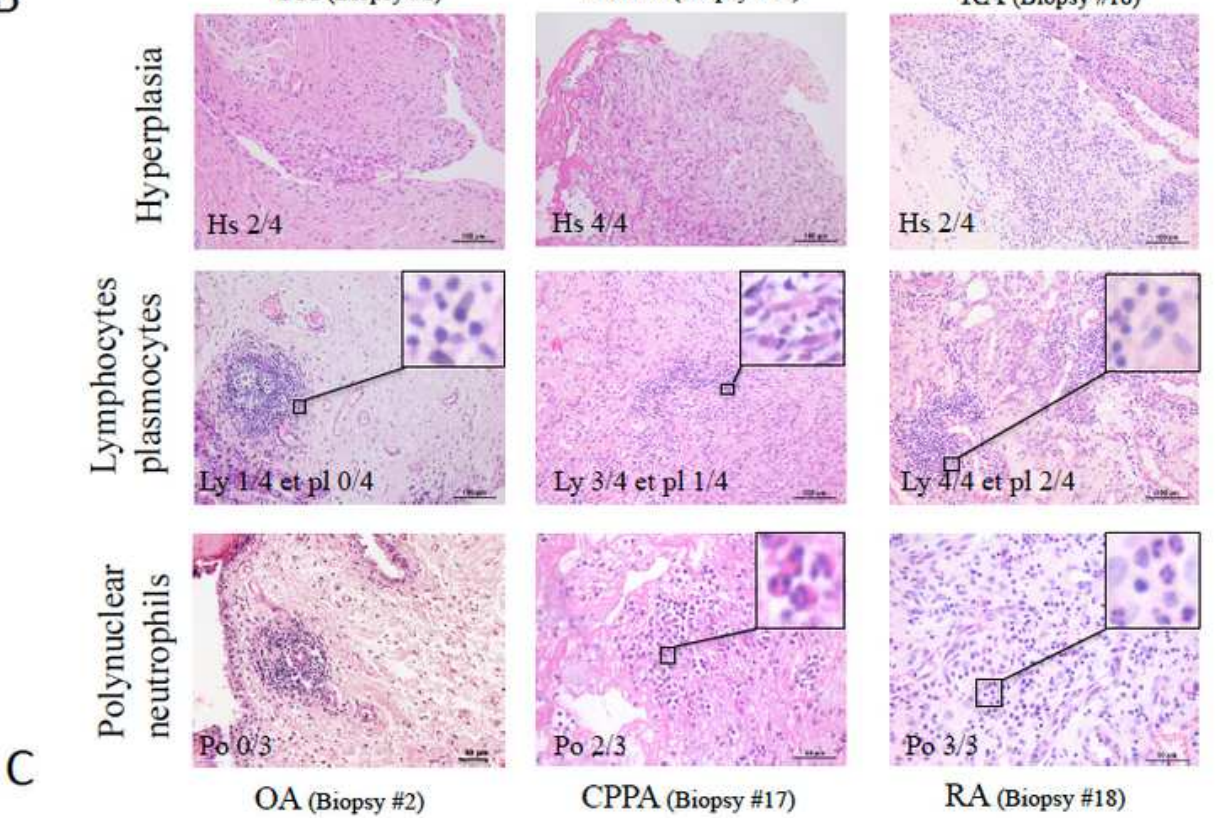

CPPA (Biopsy \#17)

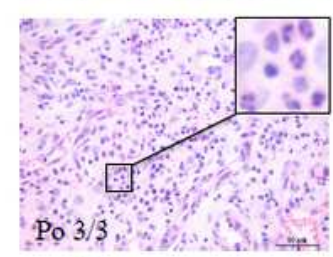

RA (Biopsy \#18)

ڤ̊

CD68 - 2/3

$\mathrm{CD} 68-3 / 3$

CD68 3/3

\section{Figure 1}

Histological scoring of synovial biopsies A) Classification of OA, CPPA and RA synovial biopsies $(n=24)$ according to the histological score based on the following criteria: synovial hyperplasia (hs, $0-4$ ), infiltration of lymphocytes (ly, $0-4)$, plasmocytes (pl, $0-4)$, polynuclear neutrophils (po, $0-3)$ and 
macrophages (CD68, $0-3)$. B) Histological representation of hematoxylin eosin stained sections for synovial hyperplasia and infiltration of lymphocytes/plasmocytes and polynuclear neutrophils in one OA, CPPA or RA biopsy. C) Immunohistochemistry using anti-CD68 antibody showing macrophage infiltration in OA, CPPA and RA synovial biopsies.

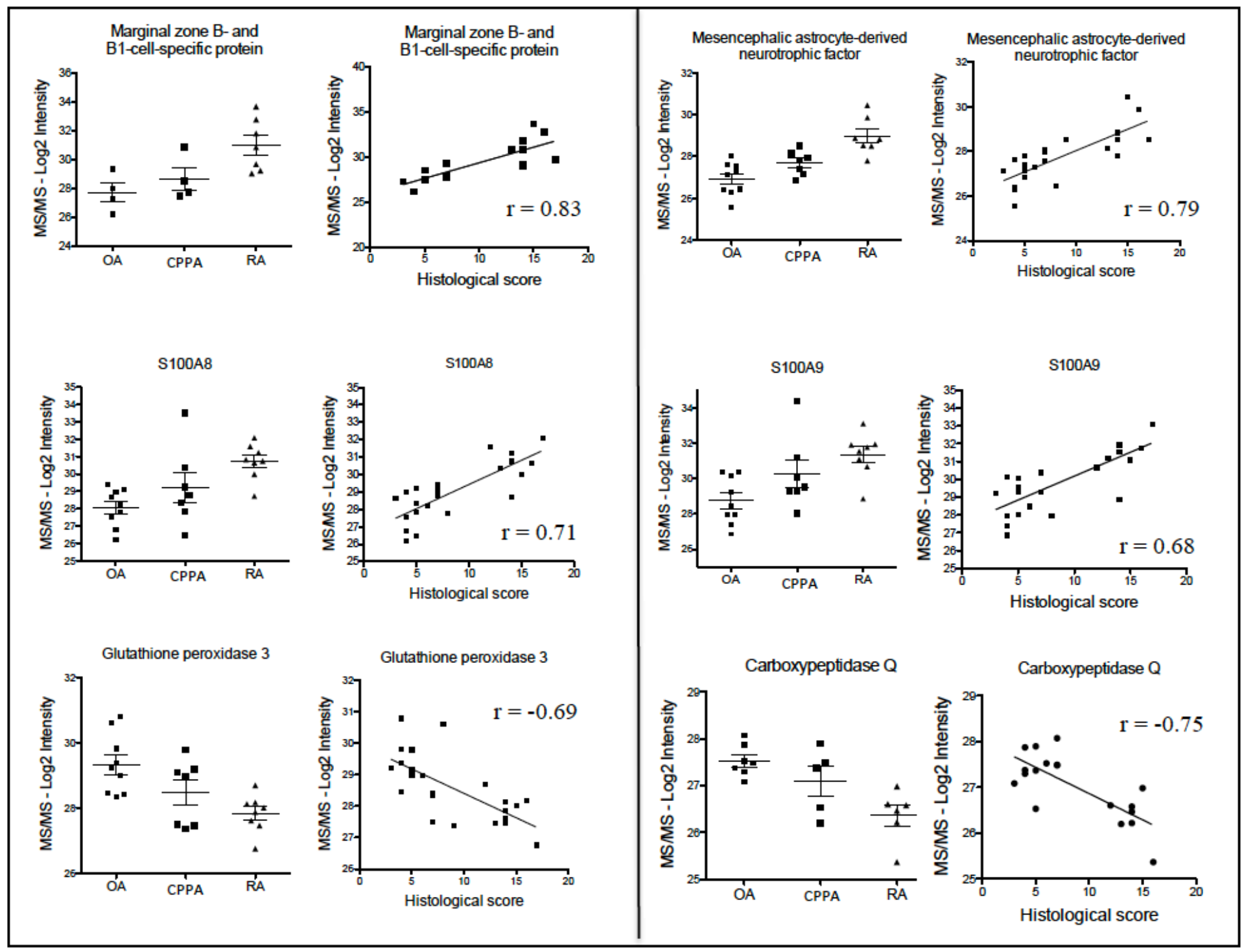

Figure 2

Distribution of protein intensities among the 3 groups (OA, CPPA and RA) and correlation with the histological score Illustration of some proteins from Table 2 for which log2 intensities obtained by MS/MS are represented among the three groups (OA, CPPA and RA) and statistically correlated to the histological score. 
A

\begin{tabular}{|l|r|rrrrrr}
\hline Gene functional classification & & & & & & & \\
\hline Pathways & Genes & $\%$ & PValue & Bonferroni & Benjamini & FDR \\
\hline Protein processing in endoplasmic reticulum & 11 & 21.57 & $1.21 \mathrm{E}-09$ & $7.72 \mathrm{E}-08$ & $7.72 \mathrm{E}-08$ & $1.23 \mathrm{E}-06$ \\
\hline
\end{tabular}

B

\begin{tabular}{|l|l|}
\hline \multicolumn{2}{|l|}{ Protein processing in endoplasmic reticulum } \\
\hline ID & Gene Name \\
\hline 51726 & DnaJ heat shock protein family (Hsp40) member B11 (DNAJB11) \\
811 & calreticulin (CALR) \\
10961 & endoplasmic reticulum protein 29 (ERP29) \\
23193 & glucosidase II alpha subunit (GANAB) \\
7184 & endoplasmin or glucose-related protein 94 - GRP94 (HSP9OB1) \\
3303 & heat shock protein family A (Hsp70) member 1A/1B (HSPA1A) \\
3309 & 78 kDa glucose-related protein - GRP78 or BiP (HSPA5) \\
10525 & hypoxia up-regulated 1 - GRP170 (HYOU1) \\
3998 & lectin, mannose binding 1 (LMAN1) \\
9601 & protein disulfide isomerase family A member 4 (PDIA4) \\
81567 & thioredoxin domain containing 5 (TXNDC5) \\
\hline
\end{tabular}

C

D
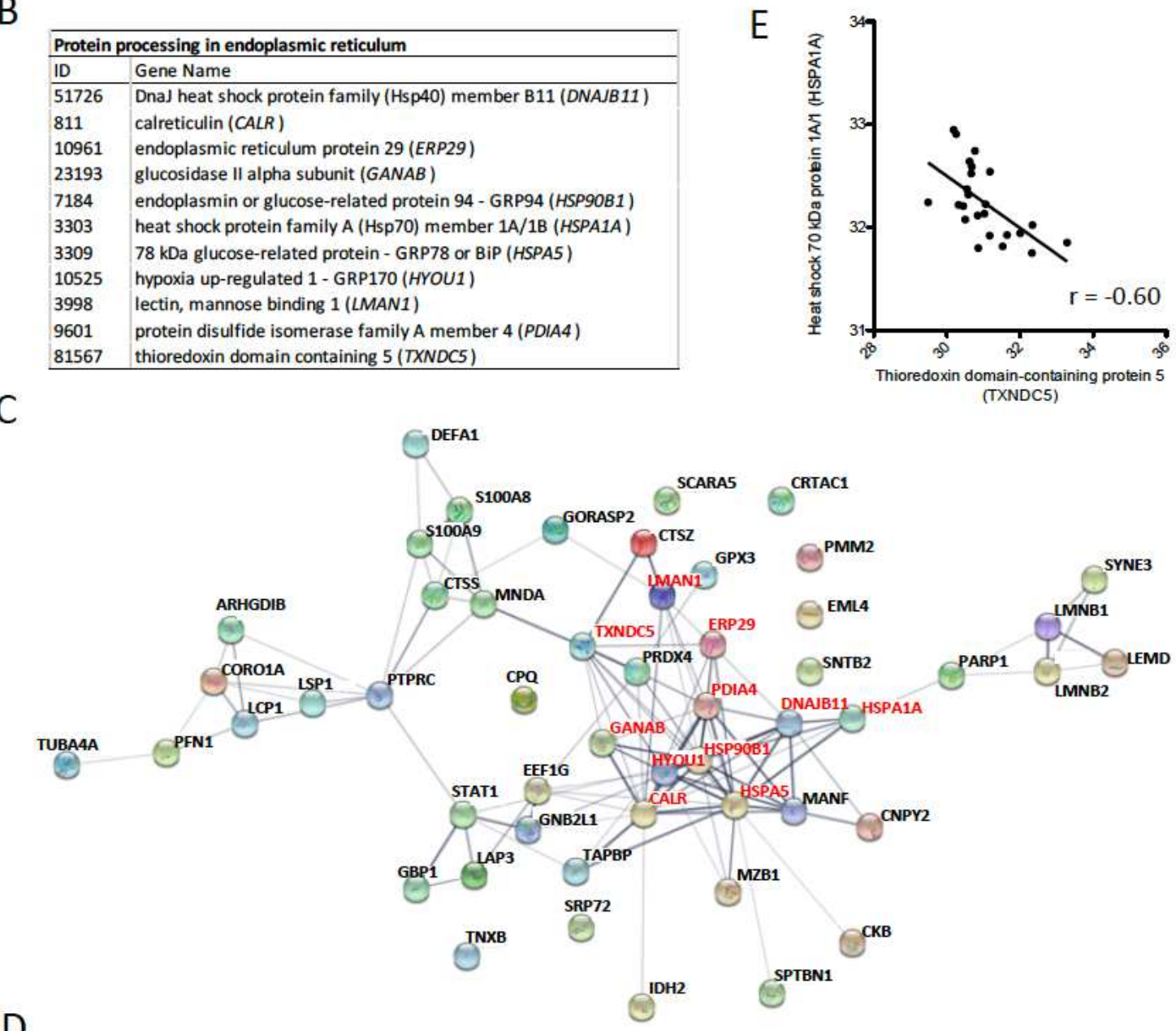

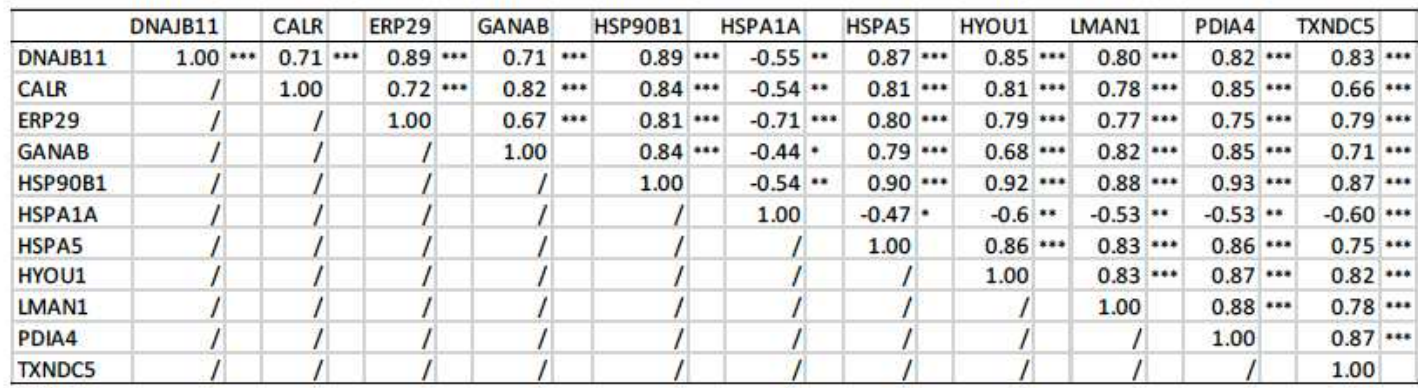

\section{Figure 3}

ER stress proteins detected in the inflamed synovial membrane: A) DAVID analysis performed on the 51 biomarkers highlighted for their significant correlation to the histological score, for their functional classifications. The pathway entitled "protein processing in endoplasmic reticulum (ER)" was selected. B) Proteins involved in the ER pathway according to DAVID analysis. C) STRING protein-protein interaction among the 51 proteins highlighted in Table 2. Red writings indicate proteins involved in the ER network 
according to DAVID analysis. D) Correlation parameters between the 11 proteins involved in the ER according to DAVID. E) Negative correlation between HSPA1A and TXNDC5 protein levels.

\section{Supplementary Files}

This is a list of supplementary files associated with this preprint. Click to download.

- Additionalfile1.pdf 